\title{
Uterine Leiomyosarcoma Presenting With Bilateral Orbital and Left Neck Metastases
}

\author{
Sunil Dutt Sharma ${ }^{\mathrm{a}, \mathrm{b}}$, Gaurav Kumar ${ }^{\mathrm{a}}$, Hesham Kaddour ${ }^{\mathrm{a}}$
}

\begin{abstract}
Uterine leiomyosarcoma is a rare neoplasm of mesodermic origin with a predilection for hematologic dissemination, but bilateral orbital metastases have never been reported. A 63-year-old lady presented with an 18-month history of a left neck and left breast mass, and a short history of left eye pain and intermittent diplopia. Ultrasoundguided core biopsy of the left neck node showed evidence of leiomyosarcoma, and computed tomography of the orbits demonstrated bilateral infraorbital masses arising from the medial recti. The patient was diagnosed with uterine leiomyosarcoma with orbital metastases. She was treated with bilateral orbital radiotherapy but had further disease progression, and has since started doxorubicin. Surgery is usually advocated in the management of metastatic leiomyosarcoma, but given the extensive metastases in this case, radiotherapy was the preferred option. This is the first reported case of bilateral orbital and neck metastases from a uterine leimyosarcoma.
\end{abstract}

Keywords: Leiomyosarcoma; Neoplasm metastases; Orbit

\section{Introduction}

Leiomyosarcoma is a rare malignant tumor of smooth muscle origin that has a predilection for hematological spread [1]. It is most commonly found in the uterus, stomach, small intestine, vasculature and retroperitoneum [2]. It usually remains dormant for many years and then can recur.

Leiomyosarcomas are generally resistant to radiotherapy and chemotherapy and there is a general opinion that aggressive surgery with wide resection margins is the treatment of choice [3].

Whilst primary leiomyosarcoma in the orbit has been re-

Manuscript accepted for publication February 06, 2015

aDepartment of Otorhinolaryngology, Queens Hospital, Rom Valley Way, Romford, Essex RM7 0AG, UK

${ }^{b}$ Corresponding Author: Sunil Sharma, Department of Otorhinolaryngology, Queens Hospital, Rom Valley Way, Romford, Essex RM7 0AG, UK.

Email: sunilsharma@doctors.org.uk

doi: http://dx.doi.org/10.14740/jmc2071w ported by several authors, there are only three previously documented case reports of orbital metastases of leiomyosarcoma from a uterine primary [4-7]. However, there are no documented cases of bilateral orbital metastases from the uterus. Here we present a case of a middle-aged lady with bilateral orbital and neck metastases from a primary uterine leiomyofibroma.

\section{Case Report}

A 63-year-old Caucasian lady presented to the ENT department with an 18-month history of a left-sided neck lump and left breast lump. She also complained of a 2-week history of left eye pain and diplopia.

Physical examination demonstrated a well-circumscribed round, fixed $3 \mathrm{~cm}$ posterior triangle lymph node on the left side of the neck. There was also a left breast lump about $3 \mathrm{~cm}$ in diameter. Eye examination demonstrated a slight proptosis of the left eye, but no diplopia. There was a restriction in elevation of the right eye. The visual acuity was 0.06 in the right eye and 0.30 in the left eye. The intraocular pressure was $14 \mathrm{~mm} \mathrm{Hg}$ in the right eye and $13 \mathrm{~mm} \mathrm{Hg}$ in the left eye, with exophthalmic radius of $23 \mathrm{~mm}$ in each eye.

Ultrasound-guided core biopsy of the left neck node demonstrated histological features of leiomyosarcoma with intersecting and intertwining bundles of elongated spindle cells with abundant deeply eosinophilic cytoplasm with a hemangiopericytomatous pattern focally. The nuclei were markedly pleomorphic with focal ischemia and caseation necrosis. The tumor cells expressed SMA and desmin but not CD34 or S100. There was a Ki-67 index of up to $19 \%$.

Computed tomography of the orbits demonstrated bilateral masses arising from the medial orbit and replacing the medial rectus muscle, larger on the left side $(10 \mathrm{~mm})$ than on the right side (Fig. 1). Biopsy was performed of the left orbital mass which confirmed metastatic deposits on histology from leiomyosarcoma.

The patient was urgently referred to the tertiary sarcoma center where she had a positron emission tomography-computed tomography (PET-CT) scan that demonstrated the primary in the uterus. Histological analysis of the uterine mass confirmed primary uterine leiomyosarcoma.

Given the extensive disease, the decision was made to treat it with bilateral orbital radiotherapy (30 Gy in 10 frac- 


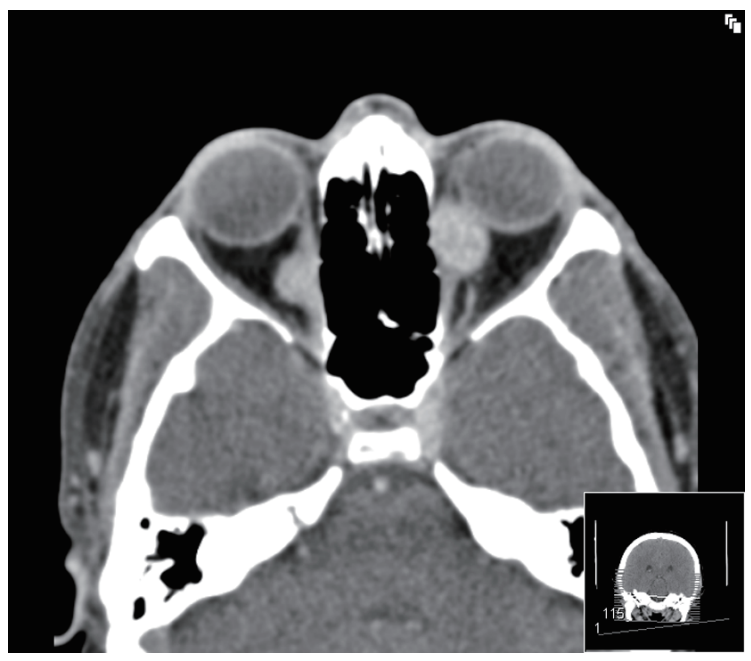

Figure 1. Axial section from CT orbits of patient demonstrating bilateral $1 \mathrm{~cm}$ diameter homogenous soft tissue lesions on the inner side of the extraconal space arising from the medial recti, representing orbital metastases from a primary uterine leiomyosarcoma.

tions). Unfortunately there was further disease progression and she has since had six cycles of doxorubicin as part of the GeDDiS trial [8]. The patient has now finished her course of treatment, and now is for palliative treatment.

\section{Discussion}

Leiomyosarcoma is a smooth muscle neoplasm, typically in the uterus, gastrointestinal tract, retroperitoneum and vascular tissue [2]. However, it can be found in other parts of the body in mesenchymal cells with smooth muscle differentiation potential [2]. They typically occur in middle-aged to older women with an average age of diagnosis of 64 years (range 36 - 84 years) [9].

Leiomyosarcoma makes up around $1 \%$ of all uterine malignancies with an incidence of 0.64 per 100,000 women [1]. The risk of metastases ranges from $45 \%$ to $73 \%$, most commonly to the lungs, liver and peritoneal cavity [1].

Metastases of leiomyosarcoma to the head and neck region are very rare, and only a few cases have been reported, including the mandible, temporalis muscle, larynx, trachea, external auditory canal and middle ear $[10,11]$.

Orbital leiomyosarcomas are extremely rare [3]. A search of MEDLINE (1966 - 2013) using the keywords "uterine leiomyosarcoma" and "orbit" produced 15 citations. There are four reported cases of metastatic leiomyosarcoma of the orbits from a uterine primary. Drake and Dobben described the case of a 52-year-old woman with right retro-orbital leiomyosarcoma who was diagnosed with primary uterine leiomyosarcoma 20 years prior to presentation with the orbital symptoms [6]. She unfortunately died of widespread metastases. Gardner described a 55-year-old case with metastasis to the right retrobulbar region, who died 4 years post diagnosis [7]. Su et al described the case of a 55-year-old lady with leiomyosarcoma of the uterus with sphenoid bone and orbital metastases, who was treated with aggressive surgery and survived at 10 months follow-up [5]. Kaltreider et al have described the case of a 43-year-old lady with leiomyosarcoma of the medial orbit, with a previous history of buttock and uterine leiomyosarcoma, who survived at 8 months follow-up.

There are also reported cases of secondary leiomyosarcoma invading the orbit from the paranasal sinuses and orbital leiomyosarcoma as a complication of orbital radiation for retinoblastoma $[12,13]$. However, a thorough literature review reveals that there are no reported cases of primary uterine leiomyosarcoma presenting with bilateral orbital, left neck and left breast metastases.

Women with advanced uterine leiomyosarcoma have very poor 5 -year survival rates ranging from $0 \%$ to $42 \%$ [14]. Surgery is generally recommended, even in metastatic leiomyosarcoma, as it tends to have a poor response to chemoradiotherapy, and indeed there have been survival benefits reported when there is metastatic disease in the brain or lungs [14, 15].

Soft tissue sarcoma has been reported to be best treated with complete surgical excision with $1 \mathrm{~cm}$ margins when surgery alone is used, and $0.5 \mathrm{~cm}$ margins if a combination of surgery and radiotherapy is used [16]. There are, however, no randomized control trials for treatment of soft tissue sarcomas [11]. There are some prospective trials that have shown clear benefits of adjuvant radiotherapy in soft tissue sarcomas [17, 18]. More recent studies have demonstrated benefits of chemoor radiotherapy alone in treating leiomyosarcoma, which has implications for future treatment [2].

The decision to proceed with surgery in the context of metastatic leiomyosarcoma is dependent on many factors, not least the informed patient's wishes. In the case reported, given the extensive disease, the patient elected for radiotherapy given the risk to sight of bilateral surgery.

When orbital leiomyosarcoma is considered, the differential should include primary, secondary, metastatic and radiation induced tumor. The importance of a thorough gynecological history cannot be emphasized enough in these patients, as the uterus is a relatively common site for leiomyosarcoma. Increasingly, there are more options in the treatment of leiomyosarcoma, including surgery, chemo- or radiotherapy. Given the rarity of the condition, long-term survival is difficult to predict, but close follow-up is always necessary.

\section{Conclusion}

Uterine leiomyosarcoma is a rare malignant tumor of smooth muscle origin that has a predilection for hematologic dissemination.

Most metastatic lesions occur in the lung and abdomen whilst involvement of the orbit is rare.

We describe a case report of a 63 -year-old lady presenting with bilateral orbital metastases from a primary uterine leiomyosarcoma, which has not been reported before.

The decision to proceed with surgical treatment in the setting of metastatic disease must be individualized, but in this case as the disease was so advanced, chemoradiotherapy was the treatment of choice. 
This case highlights the need for a careful, thorough history and examination when faced with the rare presentation of orbital leiomyosarcoma.

\section{Acknowledgement}

Grateful thanks to the administrative staff in the ENT Department in Queens Hospital. This case was presented at the Sixth World Congress for the Brain, Skull Base and Spine in Milan, Italy in April, 2014.

\section{Financial Support}

This research received no specific grant from any funding agency, commercial or not-for-profit sectors.

\section{Conflict of Interest}

None.

\section{References}

1. Giuntoli RL, 2nd, Metzinger DS, DiMarco CS, Cha SS, Sloan JA, Keeney GL, Gostout BS. Retrospective review of 208 patients with leiomyosarcoma of the uterus: prognostic indicators, surgical management, and adjuvant therapy. Gynecol Oncol. 2003;89(3):460-469.

2. Skubitz KM, D'Adamo DR. Sarcoma. Mayo Clin Proc. 2007;82(11):1409-1432.

3. Chen J, Wei R, Ma X. Orbital metastasis of retroperitoneal leiomyosarcoma. Med Oncol. 2012;29(1):392-395.

4. Kaltreider SA, Destro M, Lemke BN. Leiomyosarcoma of the orbit. A case report and review of the literature. Ophthal Plast Reconstr Surg. 1987;3(1):35-41.

5. Su GW, Hong SH. Leiomyosarcoma of the uterus with sphenoid bone and orbital metastases. Ophthal Plast Reconstr Surg. 2007;23(5):428-430.
6. Drake ET, Dobben GD. Leiomyosarcoma of the uterus with unusual metastases. J Am Med Assoc. 1959;170(11):1294-1298.

7. Gardner LU. A Case of Metastatic Leiomyosarcoma Primary in the Uterus. J Med Res. 1917;36(1):19-30 13.

8. http://public.ukcrn.org.uk/search/StudyDetail. aspx?StudyID=8444.

9. Lin IC, Wu CT, Liao SL, Lin LL. Primary orbital leiomyosarcoma. Ophthal Plast Reconstr Surg. 2005;21(6):451453.

10. Kang J, Levinson JA, Hitti IF. Leiomyosarcoma of the parotid gland: a case report and review of the literature. Head Neck. 1999;21(2):168-171.

11. Aslan E, Kuzeyli K, Cakir E, Reis A. Temporalis muscle metastasis of the uterine leiomyosarcoma: a case report. Turk Neurosurg. 2008;18(2):215-218.

12. Jakobiec FA, Mitchell JP, Chauhan PM, Iwamoto T. Mesectodermal leiomyosarcoma of the antrum and orbit. Am J Ophthalmol. 1978;85(1):51-57.

13. Folberg R, Cleasby G, Flanagan JA, Spencer WH, Zimmerman LE. Orbital leiomyosarcoma after radiation therapy for bilateral retinoblastoma. Arch Ophthalmol. 1983;101(10):1562-1565.

14. Dinh TA, Oliva EA, Fuller AF, Jr., Lee H, Goodman A. The treatment of uterine leiomyosarcoma. Results from a 10-year experience (1990-1999) at the Massachusetts General Hospital. Gynecol Oncol. 2004;92(2):648-652.

15. Vaquero J, Martinez R, el Barkani A, Gomez-Angulo JC, Escandon J. Leiomyosarcoma metastatic to the brain with prolonged survival. J Neurosurg Sci. 1989;33(3):291292.

16. Eilber FR, Eckardt J. Surgical management of soft tissue sarcomas. Semin Oncol. 1997;24(5):526-533.

17. Pisters PW, Harrison LB, Leung DH, Woodruff JM, Casper ES, Brennan MF. Long-term results of a prospective randomized trial of adjuvant brachytherapy in soft tissue sarcoma. J Clin Oncol. 1996;14(3):859-868.

18. Suit HD, Mankin HJ, Wood WC, Gebhardt MC, Harmon DC, Rosenberg A, Tepper JE, et al. Treatment of the patient with stage M0 soft tissue sarcoma. J Clin Oncol. 1988;6(5):854-862. 